\title{
Preference Constraints: New Global Soft Constraints Dedicated to Preference Binary Relations
}

\author{
Rémy-Robert Joseph ${ }^{1}$, Peter Chan ${ }^{2}$, Michael Hiroux ${ }^{1,2}$, Georges Weil ${ }^{1,2}$ \\ ${ }^{1}$ Université Joseph Fourier-Grenoble 1, Laboratoire IMAG/TIMC, Equipe SIC, \\ Institut Albert Bonniot, 38706 La Tronche, France \\ ${ }^{2}$ Equitime S.A., Grand sablon, 4 Avenue de l'Obiou 38700 La Tronche, France \\ Remy.Joseph@imag.fr
}

\begin{abstract}
In this article, we propose a new soft constraint called preference constraint, squaring well with the decision theory concept of preference binary relation. We show how to use it for designing complex hierarchical preference information based on preference binary relations for combinatorial problems. Finally, preference-based constraint systems are defined and associated best quality choice problems are introduced. This new model offers greater flexibility to represent and make complex decisions with computers.
\end{abstract}

\section{Introduction}

Until now, the privileged preference representation used for combinatorial problems has been the objective function. It is exclusively used at every aggregation level [7] of a hierarchical preference model, and has remarkable structural properties as transitivity and completeness. These properties are often judged too restrictive, because some important aggregation concepts are incomplete by definition, as efficiency, unanimity, equity [10], to quote only few of them. Attributes cannot be necessarily transitive because of uncertainty [12]. Obviously these properties are desirable for a collective choice, but we shall not make a fetish of them [10]. For all these reasons which are the rule in practical works, it is necessary to enlarge objective function-based preference models toward weakly structured aggregation rules. In this work, we extend preference models to aggregation rules based on preference binary relations. Often used in multi-criteria decision aiding (MCDA) and social choice theory (SCT)』, for their abilities of preference modelling and decision aiding, preference binary relations stay yet almost non-existent in combinatorial (and continuous) optimization2. Both interactivity and weaker preference models ("bounded" rationality) are necessary to improve the decision-making.

${ }^{1}$ MCDA [12] attends to evaluation of practical problems having solutions given in extension, and SCT [10] points out theoretical works on the characterization of adequate aggregation rules for collective and public problems.

2 Note nevertheless that multi-objective mathematical programming (MOMP) has allowed going beyond classical optimization models by admitting the transitive incomplete Pareto

A. M. Frisch (Ed.): CP 2003, $2^{\text {nd }}$ International Workshop MRCSP, p. 104-109, 2003 
This article is outlined as follows: After a review of basic notions in constraint programming in section 2, we introduce preference constraints as a way to represent preference binary relations (section 3 ). The $4^{\text {th }}$ section is devoted to preference binary relations obtained from aggregation rules for which preference constraints are adapted. Finally, preference-based constraint systems are introduced in 5 .

\section{Background in Constraints}

A finite constraint system $C S$ is defined as a set of $n$ variables $V=\left\{v_{1}, \ldots, v_{n}\right\}$, a finite domain $D(v)$ of possible values for each variable $v \in V$, and a set of $m$ constraints $C=\left\{c_{1}, \ldots, c_{m}\right\}$ among variables. A constraint $c$ is characterized by a set of variables $V(c) \subseteq V$ and a feasible solution set $S(c)$ included in the Cartesian product of domains associated to $V(c): S(c) \subseteq \mathcal{D}_{V(c)}=X_{v \in V(c)} D(v)$. An element of $\mathcal{D}_{V(c)}$ is called a solution of $c$, and an element of $\mathcal{D}_{V}=D\left(v_{1}\right) \times \ldots \times D\left(v_{n}\right)$ is called a solution of the constraint system CS. A value $d$ of a variable $v(\Leftrightarrow d \in D(v))$ is consistent with a constraint $c$ iff $v \notin V(c)$, or there exists a feasible solution $x$ of $c$ such that $x(v)=d$. Otherwise, $d$ of $v$ is said inconsistent with $c$. As from this definition, it is possible to define different kind of consistency properties on variables, constraints and constraint systems 3 . A variable $v$ is consistent with a constraint iff $D(v)$ is not empty and all its values are consistent with $c$. A constraint $c$ is globally inverse consistent iff for all $v$ in $V(c), v$ is consistent with $c$. Given a constraint $c$ and a set of domains $D$ associated to variables $V(c)$, a filtering algorithm for $c$ is an algorithm establishing a consistency level for $c$. A constraint provided with an adjusted filtering algorithm is called a global constraint ([9], [8]).

A combinatorial constraint satisfaction problem (CSP) on a given finite constraint system $C S=(V, D, C)$ is concerned with the search for an element $x$ of $\mathcal{D}_{V}$ such that for all constraints $c \in C$, the projection 4 of $x$ on $V(c)$ is consistent with $c$. Such a solution $x$ is called a feasible solution of the constraint system and the set of all feasible solutions of a constraint system $C S$ is noted $S_{\mathrm{CSP}}(C S)$.

\section{Preference Binary Relations and Preference Constraints}

After defining the preference binary relation, we present an adequate model to represent preference binary relations on constraint systems (i.e. Cartesian product sets).

dominance as final aggregation rule. Others as the lexicographic, the maximin and the leximin rules, have been used to synthesize objective functions. Although transitive and complete these aggregation rules are not representable by objective functions (see references given in [10]).

${ }^{3}$ For further information, see [11], [2], among others.

${ }^{4}$ Projection of $x \in \mathcal{D}_{V}$ on $V 1 \subseteq V$ is the element $x_{1}$ of $\mathcal{D}_{V 1}$ such that $x_{1}(v)=x(v) \forall v \in V 1$. 


\subsection{Background in Decision Theory}

There exists different ways of modelling preferences [12]. This article is devoted to preference binary relations, defined here:

A binary relation $\succcurlyeq$ on a set $S$ is a subset of the Cartesian product $S \times S$. We will note here $x \geqslant y$ instead of $(x, y) \in \geqslant$, and not $(x \geqslant y)$ to designate $(x, y) \notin \geqslant$.

Given a set of solutions $S$, a preference binary relation $\succcurlyeq$ of an individual on $S$ is a reflexive binary relation on $S(\Leftrightarrow x \geqslant x$, for all $x \in S$ ) traducing the judgments of this individual concerning his preferences between the pairs of solutions. The assertion " $x$ $\succcurlyeq y$ " means " $x$ is at least as good as $y$ for the considered individual" for any solutions $x$ and $y$ of $S$. A preference binary relation $\succcurlyeq$ makes a partition of $S \times S$ into four fundamental binary relations called fundamental attitudes. Here is their definition:

(indifference) $\quad x \simeq y \Leftrightarrow(x \geqslant y$ and $y \geqslant x)$ for any $x, y \in S$

(strict preference) $x \succ y \Leftrightarrow(x \succcurlyeq y$ and $\operatorname{not}(y \succcurlyeq x))$ for any $x, y \in S$

(strict aversion) $\quad x \prec y \Leftrightarrow y \succ x$ for any $x, y \in S$

(incomparability) $x \| y \Leftrightarrow(\operatorname{not}(x \geqslant y)$ and $\operatorname{not}(y \geqslant x))$ for any $x, y \in S$

A preference binary relation can be also interpreted as a mapping from $S \times S$ to $A F=$ $\{\simeq, \succ, \prec, \|\}$ with $A F$ the set of fundamental attitudes. The set $P R(A F)$, made up of elements of the power set of $A F$ different from the empty set and $A F$, is called the set of attitudes.

\subsection{Preference Modelling, Soft Global Constraints and Preference Constraints}

In an explicit solution set environment, preferences are often explicitly represented [12]. But the implicit formulation of solutions $\mathcal{D}_{V}$ and feasible solutions $S_{\mathrm{CSP}}(C S)$ makes this way of modelling inconceivable. In constraint programming, preference representations have taken shape in soft constraints. Interesting soft constraints have been used in the frameworks of valued constraints systems and semiring-based constraint systems [1]. But they are limited to semiring structures on valuations. Otherwise, two kinds of preference models have handled global constraints: (a) property constraints dedicated to relevant basic properties which can be or not satisfied by a feasible solution and $(b)$ objective function constraints devoted to objective functions by way of constraints. Such soft constraints are called soft global constraints ([8], [9]). To fill the gap about soft global constraints dedicated to preference binary relations, we present the preference constraints:

A preference binary relation $\succcurlyeq$ can be described by a set of constraints $c_{\succcurlyeq}[\alpha, x]$ parameterized by a solution $x$ and an attitude $\alpha$. By noting $V$ the variable set and $D$ the domain set on which scope the constraints $c_{\succcurlyeq}[\alpha, x]$, then the set $\left\{c_{\succcurlyeq}[\alpha, x], \forall(\alpha, x) \in\right.$ $\left.P R(A F) \times D_{V}\right\}$ is called the preference constraint associated to the preference binary relation $\succcurlyeq$. For short, we will note $\left\{c_{\succcurlyeq}[\alpha, x]\right\}_{\alpha, x}$.

The feasible set of $c_{\succcurlyeq}[\alpha, x]$ is noted $S\left(c_{\succcurlyeq}[\alpha, x]\right)=\left\{y \in \mathcal{D}_{V}\right.$ such that: $\left.y \alpha \succcurlyeq x\right\}$, with $\alpha \succcurlyeq$ indicating the attitude $\alpha$ of the preference binary relation $\succcurlyeq$. In a digraph context, $S\left(c_{\succcurlyeq}[\alpha, x]\right)$ describes the neighborhood of $x$ in the set $\mathcal{D}_{V}$ according to the binary relation $\alpha$. This modelling of the preference binary relation offers large perspectives in solving problems, as we will see in the following. 


\section{Aggregation and Preference Constraints}

We show here possibilities offered by preference constraints in the building of complex hierarchical preference models.

\subsection{Aggregation Rules and Preference Models}

In complex real world problems, the evaluation of solutions can be done from several persons or/and from several viewpoints for each person. This preference information is methodically synthesized with several aggregations rules ([7], [3]) in order to obtain a collective preference binary relation representing the preference model of the problem.

Here, the term individual designates a human, a group, a society or someone's viewpoint; and $I=\{1, \ldots, n\}$ points out a set of individuals. From now pref(i, $S$ ) refers to either the objective function $f_{i}$ or the preference binary relation $\succcurlyeq_{i}$ of the individual $i$ on the set $S$. The component of a preference model, allowing to synthesize preferential information, is the aggregation rule. An aggregation rule is a functional relation $A R$ such that for any set of $n$ individual preferences $\operatorname{pref}(1, S), \ldots$, $\operatorname{pref}(n, S)$ (one for each individual), one and only one collective preference $\operatorname{pref}(I, S)$ is determined, $\operatorname{pref}(I, S)=A R(\operatorname{pre} f(1, S), \ldots, \operatorname{pref}(n, S))$.

As examples we mention: the weighted sum function ([4], [7], [12]), the majority method ([10], [12]) and the lexicographic rule ([4], [10], [12]).

\subsection{Preference Constraints for Aggregation Rules.}

The semantics of a preference constraint can be defined as an aggregation rule allowing preference binary relations and objective functions as individual preferences and a binary relation as collective preference. To each aggregation rule $A R$ is associated one preference constraint noted $\left\{c_{A R}[\alpha, z]\right\}_{\alpha, z}$ or $\left\{c_{\succcurlyeq}[\alpha, z]\right\}_{\alpha, z}$, if $\succcurlyeq$ is the collective preference binary relation of $A R$. Individual preferences are noted $\left\{c_{i}[\alpha\right.$, $z]\}_{\alpha, z}$ for any individual $i \in I=\{1, \ldots, n\}$. The variable set of $\left\{c_{A R}[\alpha, z]\right\}_{\alpha, z}$ is equal to the union of individual preferences variable sets. Whereas the variable set, the feasible solution set of $\left\{c_{A R}[\alpha, z]\right\}_{\alpha, z}$ is parameterized by an attribute and a solution. Here is their definition:

$$
\begin{gathered}
V\left(\left\{c_{A R}[\alpha, z]\right\}_{\alpha, z}\right)=V\left(\left\{c_{1}[\alpha, z]\right\}_{\alpha, z}\right) \cup \ldots \cup V\left(\left\{c_{n}[\alpha, z]\right\}_{\alpha, z}\right) \\
S\left(c_{A R}[\alpha, x]\right)=\left\{y \in \mathcal{D}_{V(A R)} \text { such that: } y \alpha_{A R} x\right\} \quad \forall(\alpha, x) \in P R(A F) \times \mathcal{D}_{V(A R)} .
\end{gathered}
$$

with $\mathcal{D}_{V(A R)}$ the Cartesian product of domains $D(v)$ for all $v \in V\left(\left\{c_{A R}[\alpha, z]\right\}_{\alpha, z}\right)$ and $\alpha_{A R}$ the attribute $\alpha$ associated to the collective preference of $A R$. Any filtering algorithm for $c_{A R}[\alpha, x]$ has to use only the elements $c_{i}\left[\alpha_{1}, z\right], \forall\left(\alpha_{1}, z\right) \in P R(A F) \times D_{V(A R)}$ in order to keep their generality. But for algorithmic efficiency they can be specialized.

Preference constraints devoted to aggregation rules give a recursive definition of preference constraints. They are components of a preference model. Like the cardinality operator [13], they are abstractions; which argue in favor of their modelling power. 


\section{Preference-Based Combinatorial Choice Problems}

The instance of a combinatorial problem is made up of two parts. The first one, the feasibility model, describes feasible solutions by way of constraints and variables. The second one, the preference model, describes all the information necessary to compare solutions (different actors' viewpoints, etc). Preference constraints can be used to design the hierarchical preference model of some constraint-based choice problem instances. Thus, a preference-based constraint system is a couple $(C S,\{c \succcurlyeq[\alpha$, $z]\}_{\alpha, z}$ ), where $C S$ is a constraint system describing the set of solutions and feasible solutions, and $\{c \succcurlyeq[\alpha, z]\}_{\alpha, z}$ is a preference constraint possibly defined recursively.

Several choice problems can be defined from a preference-based constraint system. For example a problem searching one best quality solution and giving some indications on the quality of the returned solution (optimality, maximality or only feasibility):

Preference-based combinatorial constraint search problem (P-CCSP): Given a preference-based constraint system $\left(C S,\left\{c_{\succcurlyeq}[\alpha, z]\right\}_{\alpha, z}\right)$, returns one optimal solution $x\left(\Leftrightarrow x \in S_{\mathrm{CSP}}(C S)\right.$ and $\left.\forall y \in S_{\mathrm{CSP}}(C S), x \geqslant y\right)$ with the label “optimal”, if such a solution exists, else returns one maximal solution $x\left(\Leftrightarrow x \in S_{\mathrm{CSP}}(C S)\right.$ and $\forall$ $\left.y \in S_{\mathrm{CSP}}(C S), \operatorname{not}(y \succ x)\right)$ with the label "maximal”, if such a solution exists, otherwise returns a feasible solution with the label "feasible", if such a solution exists, else returns "no".

Partial problems can be defined from P-CCSP, by only returning for example one maximal solution or else "no", etc. Next, these problems can be specialized according to properties of $\succcurlyeq$. In this way, the partial preorder-based combinatorial constraint search problem (PPO-CCSP) is defined. This latter problem returns either an optimal solution, or a maximal solution, or "no", because the existence of a feasible solution certifies at least the existence of a maximal solution (see [12]).

Solving a preference-based combinatorial problem is not limited to finding one maximal solution [12], if such solutions exist. It's necessary, in the general case, to propose algorithmic tools exploring the whole maximal (or optimal) set. Interactive tools are very well adapted for these kinds of tasks. Sometimes, when we have any guaranty on the size of such a set, and that the problem ventures to do it, the problem of generating all solutions of a whole maximal set can be envisaged. We call this enumerating version of P-CCSP, the preference-based combinatorial constraint choice problem (P-CCP). In the same way, the specialized version PPO-CCP can be defined.

A great amount of work have been carried on the search for a maximal solution of a transitive preference binary relation $\succcurlyeq$ by way of an objective function (see [4], [12], for review). For this goal, it is necessary to identify some objective functions having their optimal set included in this of $\left(S_{\mathrm{CSP}}(C S), \succcurlyeq\right)$. On the other hand, global constraints give us the possibility to build a complex instance and then to solve it, without going through this theoretical identification. This possibility of customization of the preference models opens great perspectives. Recently, Gavanelli [5] presented two Branch-and-Bound-based algorithms solving the PPO-CCP, by using the particular case of $\alpha=\{\succ, \|\}$ of preference constraints. Afterwards, he designed a 
filtering algorithm for this partial preference constraint associated to the Pareto dominance aggregation rule [6].

\section{Conclusions and Perspectives}

This article gives a general framework to design and solve by way of constraint programming, combinatorial problems allowing complex preference models based on preference binary relations. It allows designing preference binary relations at an individual, intermediary and global level in preference models, conceding thus more importance to preference elicitation. Thus new soft global constraints, called preference constraints, and new combinatorial choice problems, called preferencebased constraint problems, have been introduced. One future work leading from this approach is the building of filtering algorithms for different aggregation rules.

\section{References}

1. Bistarelli S., Montanari U., Rossi F., Schiex T., Verfaillie G. et Fargier H.: Semiring-Based CSPs and Valued CSPs: Frameworks, Properties, and Comparison. Constraints: An International Journal 4 (3), (1999) 199-240

2. Cooper M. C.: An Optimal $k$-Consistency Algorithm. Artif. Intelligence 41 (1989) 89-95

3. Corner J., Buchanan J., Henig M.: Dynamic Decision Problem Structuring. Journal of Multi-Criteria Decision Analysis 10 (2001) 129-141

4. Ehrgott M., Gandibleux X.: An Annotated Bibliography of Multiobjective Combinatorial Optimization. OR Spektrum 22 (2000) 425-460

5. Gavanelli M.: Interactive Constraint Satisfaction Problems for Artificial Vision. Ph.D. thesis of the University of Ferrara, Italy (2002)

6. Gavanelli M.: An Implementation of Pareto Optimality in CLP(FD). Proceedings of the CP-AI-OR'02 (2002) 49-64

7. Keeney R. L., Raïffa H.: Decisions with Multiple Objectives: Preferences and Value Tradeoffs. John Wiley \& Sons, New York (1976)

8. Régin J. C., Petit T., Bessière C., Puget J. F.: New Lower Bounds of Constraints Violations for Over-Constrained Problems. In: Walsh T. (ed.): Proceedings CP'2001. Lecture Notes in Computer Science, Vol. 2239. Springer-Verlag, Berlin Heidelberg New York (2001) 332345

9. Régin J.-C., Rueher M.: A Global Constraint Combining a Sum Constraint and Difference Constraints. In Dechter R. (ed.): Proceedings CP'2000. Lecture Notes in Computer Science, Vol. 1894. Springer-Verlag, Berlin Heidelberg New York (2000) 384-395

10. Sen A. K.: Collective Choice and Social Welfare. Series in Advanced textbooks in economics, vol. 11, Elsevier science publishers, Netherlands (1970)

11. Verfaillie G., Martinez D., Bessière C.: A Generic Customizable Framework for Inverse Local Consistency. In Proceedings of AAAI\IAAI'99, 18-22 july 1999, Orlando, Florida, USA, AAAI Press / The MIT Press (1999) 169-174

12. Vincke, P.: Multicriteria Decision-Aid. John Wiley \& Sons (1992)

13. van Hentenryck P., Deville Y.: The Cardinality Operator : A New Logical Connective for Constraint Logic Programming. Logic Programming : Proceedings of the $8^{\text {th }}$ International Conference (ICLP’91), MIT Press, Cambridge, Massachusetts (1991) 745-759 